\title{
Amigos imaginarios y la comprensión de las emociones aparentes en niños con edades entre los 3 y 6 años*
}

\section{Imaginary Friends and Understanding of Apparent Emotions in Children Between 3 to 6 Years Old}

\author{
Jaqueline Benavides Delgado** \\ ORCID: 0000-0001-8889-4317 \\ Universidad del Valle, Colombia \\ Universidad Cooperativa de Colombia
}

Recibido: 6 de octubre de 2019

Revisado: 27 de enero de 2020

Aceptado: 2 de abril de 2020

\section{Resumen}

La presente investigación tuvo como objetivo establecer si existían diferencias en tres grupos de niños $(\mathrm{N}=43$, edades entre 3 y 6 años) respecto a la comprensión de las emociones aparentes y la popularidad entre su grupo de compañeros de clase. Los niños fueron clasificados en los siguientes grupos; niños con Al (Amigos Imaginarios, $n=13$ ), niños con OP (Objetos Personificados, $n=15$ ) y niños que no presentaron ninguna de las anteriores formas de imaginación infantil $(\mathrm{n}=15)$. Se utilizaron historias basadas en Harris, et al., (1986), en donde se ejemplificaban emociones aparentes positivas en terceros y emociones aparentes negativas en sí mismos. Los análisis realizados mostraron que no existen diferencias significativas entre los tres grupos respecto a la comprensión de emociones aparentes positivas ni negativas. Los resultados obtenidos tampoco diferencian a los niños de los tres grupos respecto a la popularidad dentro de su grupo de amigos.

Palabras Clave: amigos imaginarios, habilidades sociales, popularidad, imaginación, comprensión de las emociones

Artículo de investigación. Citar como: Benavides, D. J. (2020). Amigos imaginarios y la comprensión de las emociones aparentes en niños con edades entre los 3 y 6 años. Diversitas: Perspectivas en Psicología, 16(2), 271-281. https://doi.org/10.15332/22563067.6294

** Correspondencia: Jaqueline Benavides Delgado, Investigadora. Líder del grupo Boulomai. Universidad Cooperativa de Colombia. Dirección postal: Carrera 9 \# 172- 69, Bogotá, Colombia. Correo electrónico: jaqueline.benavidesd@campusucc.edu.co 


\section{Abstract}

The aim of this research was to establish differences across three groups of children $(N=43$, aged 3 to 6 years old) regarding the understanding of apparent emotions and popularity among their group of classmates. Children were classified into the following groups: children with IF (Imaginary Friends, $n=13$ ), children with PO (Personified Objects, $n=15$ ), and children who did not show any of the previous forms of children's imagination $(n=15)$. They were tested with stories based on Harris, et al. (1986), which exemplified positive emotions in others and apparent negative emotions in themselves. The analyzes carried out showed that there are no significant differences between the three groups regarding the understanding of apparent positive or negative emotions. Results do not differentiate children from all three groups regarding popularity within their group of friends.

Keywords: imaginary friends, social skills, popularity, imagination, understanding of emotions.

\section{Introducción}

La existencia de amigos imaginarios (Al) en un número importante de niños entre los dos años y medio y los seis años, es un hecho reportado en múltiples investigaciones (e.g., Ames y Learned, 1946; Carter y Bath, 2016; Gleason, 2002; Gleason, Sebanc y Hartup, 2000; Moriguchi, Shinohara y Ishibashi, 2016; Moriguchi y Todo, 2019; Pearson et al., 2001; Svendsen, 1934; Taylor 1999). Varias hipótesis han surgido alrededor de la aparición de Al en los niños, pero la relación entre la existencia de éstos y la conducta social, ha sido ampliamente analizada (e.g., Gleason y Kalpidou, 2014; Lin, Fu, Wan, Zhou y Xu, 2018). Las razones de este interés en relacionar la existencia de Al y las conductas sociales, se basan en la hipótesis de la soledad. De tal manera se asume que los niños que no tienen amigos o están muy solos deben crear sus propios vínculos desde la imaginación.

Los resultados de estos estudios, contrario a los que esperaría encontrar, aportan una mirada diferente de los niños con Al. Desde la década de los años 70, algunos autores han mostrado la presencia de habilidades sociales muy desarrolladas en niños con Al, en especial se destaca la capacidad para interactuar con los adultos (Manosevitz, Prentice y Wilson, 1973). Otros estudios han determinado que los niños con Al tienen mayor capacidad de representar roles, seguir los juegos simulados y utilizar objetos animados en sus juegos que los niños sin Al, es decir, "tener un Al parece ser un predictor muy poderoso de que el niño llegue a jugar feliz y sea cooperativo con niños y adultos" (Taylor, Cartwright y Carlson, 1993, p. 104). En este mismo sentido se ha determinado que los niños con niveles elevados de fantasía pueden establecer relaciones más positivas con otros, a diferencia de los niños del grupo control y presentan mayor sensibilidad social, empatía y habilidades de descentración (Singer y Singer, 1990).

En relación con el comportamiento social también se ha encontrado que los niños con una imaginación elevada tienen una mayor capacidad de aplazar las recompensas, comparado con los niños con un bajo nivel de fantasía. Esto implica que un niño con una imaginación más desarrollada puede aplazar un deseo, por ejemplo que el papá le compre un helado sin que se convierta en un problema para él, comparado con el niño que no ha desarrollado esta capacidad imaginativa. Este aplazamiento de las recomenzas, también encaja con lo que otros autores han descubierto respecto a que los niños con Al utilizan sus juegos de fantasía para regular sus emociones (Gayler y Evans, 2001; Moore y Russ, 2006; Russ, 2004; Singer, 1961). Sin embargo, no todos los estudios consideran que los niños con Al presentan una mayor capacidad social (Harter y Chao, 1992; Manosevitz, Fling y Prentice, 1977). Varios autores no encontraron diferencias significativas 
entre los niños con $\mathrm{Al}$ y el grupo control respecto a esta variable social. Incluso se encontró que los niños que tenían Al eran poco populares y menos aceptados socialmente por su grupo de compañeros que aquellos sin Al. Comparativamente, los niños con Al fueron calificados por sus profesoras como menos competentes a nivel físico y social. Los niños con Al solamente se destacaron en las competencias cognitivas (Harter y Chao, 1992; Manosevitz et al., 1977).

En definitiva, las investigaciones en este campo han permitido establecer que la aparición de los Al no es, desde luego, una sintomatología de patologías aparentes, pero tampoco es claro si tener Al favorece o no las habilidades sociales, generando así un debate entorno al tema. Harter y Chao (1992) consideran que los niños con Al tienen carencias a nivel de competencias físicas y sociales y son poco populares entre su grupo de iguales. Por el contrario, otros autores defienden que los niños con Al son extrovertidos (Manosevitz et al., 1973), felices, cooperativos (Taylor et al.,1993) y creativos (Singer, 1961). Si bien no se puede determinar que los niños con Al tengan deficiencias a nivel afectivo y pertenezcan a poblaciones clínicas, tampoco es adecuado señalar que los niños con Al tengan capacidades extraordinarias, superiores o destacadas sobre otros niños. Sin embargo, resulta interesante acercarse a este tema a partir de la comprensión y de la capacidad de los niños con Al de comprender las emociones de los otros.

\section{La compresión de las emociones}

La capacidad para comprender las emociones ajenas depende para Harris (1989) de otras capacidades que se adquieren durante los primeros años: la autoconciencia, la imaginación, la distinción entre la realidad y ficción y la capacidad de fingir. Así, en primer lugar, la autoconciencia implica que los niños sean conscientes de sus estados mentales, algo que se ha reportado es capaz de hacer un niño de dos años. Esta conciencia de sus propios estados emocionales permitirá a los niños comprender el estado de los otros. Por otra parte, mediante la imaginación los niños pueden crear personajes a los que atribuyen estados mentales (Harris, 1989).
La imaginación infantil es entonces una condición que facilita la comprensión de las emociones de los demás. Como lo consideran Harris y colaboradores (1986), ¿pueden los niños comprender que las personas expresan emociones que no sienten? Estos autores estudiaron la capacidad de los niños entre 4 y 10 años para comprender las emociones aparentes en otros y diferenciarlas de las emociones reales. Ellos reportaron que los niños de 4 a 5 años podían realizar la diferenciación de las emociones aparentes de aquellas que realmente manifestaban los personajes de las historias. Estos autores utilizaron 8 historias, 4 de las cuales reflejaban emociones aparentes positivas y otras hacían referencia a las emociones aparentes negativas. Esta clasificación correspondía a la diferenciación de los motivos por los cuales los niños ocultan sus emociones. En las historias positivas existió un interés en ocultar los sentimientos para no herir al otro, mientras que en las historias negativas se ocultan los sentimientos para engañar y obtener un beneficio personal. Los resultados reflejaron que los niños entre 6 y 10 años reconocían y diferenciaban las emociones aparentes de las reales. Por el contrario, los niños de 4 y 5 años tuvieron dificultades para realizar estas diferenciaciones y cometieron un mayor número de errores. Así pues, se concluyó que los niños de 4 y 5 años mostraban dificultades para comprender las emociones aparentes y diferenciarlas de las que se expresan, aunque no sean reales.

En los estudios acerca de la comprensión de las emociones aparentes no se ha vinculado a niños que presentan una imaginación particular, como los niños que crean Al. Teniendo en cuenta esta población y las características relacionadas que se han expuesto, es pertinente estudiar la capacidad que estos niños con Al pueden mostrar para comprender las emociones aparentes y las razones que llevan a las personas a ocultarlas. Igualmente se debe considerar si los niños de 4 y 5 años con Al logran comprender las emociones aparentes mejor que aquellos niños sin Al. Por lo anterior, esta investigación tuvo como objetivo responder a las siguientes preguntas: ¿Los niños con Al presentan habilidades sociales que les hacen ser más populares entre su grupo de compañeros y sus maestros? ¿Los niños con Al diferencian con mayor facilidad 
las emociones aparentes y las reales tanto positivas como negativas?

\section{Método}

\section{Participantes}

La muestra fue de 43 niños y niñas colombianos entre 3 y 6 años. La edad y la presencia de Al u OP fueron los criterios utilizados para seleccionar a los niños en tres grupos: 13 niños y niñas entre 3 y 6 años con $\mathrm{Al}\left(M_{\text {edad }}=4\right.$ años 6 meses; Rango = 3.10 a 5.5); 15 niños entre 4 y 6 años con OP $\left(M_{\text {edad }}\right.$ $=4$ años 4 meses; Rango $=4.0$ a 6.0); 15 niños entre 4 y 6 años sin Al ni OP $\left(M_{\text {edad }}=4\right.$ años 6 meses; Rango $=4.0$ a 5.6). Los niños fueron clasificados como niños con Al, Op, o sin Al ni OP a partir de un cuestionario entregado a los padres. Se repartieron 150 cuestionarios ( 30 cuestionarios en cada jardín de infancia), de los cuales 58 fueron devueltos. De los 58 cuestionarios, clasificados según el tipo de Al y la edad de los niñas y niñas, se escogieron 43, descartando algunos donde los padres no lograron diferenciar muy bien la existencia de los Al u OP. A partir de esta información se organizaron los 43 niños en los tres grupos descritos. Todos los niños asistían a centros de educación infantil de la ciudad de Bogotá y provenían de niveles socioeconómicos medios. Todos los padres de los niños firmaron el consentimiento informado, así como las instituciones educativas que permitieron el acceso de los investigadores a sus aulas.

\section{Instrumentos}

Para llevar a cabo el estudio se utilizaron tres instrumentos. En primer lugar, se adaptó el cuestionario Imaginary Companion Questionnaire de Manosevitz y colaboradores (1973). Este cuestionario se repartió a todos los padres de los niños para que determinaran la presencia de amigos imaginarios (Al) u objetos personificados (OP) y autorizaran la participación de sus hijos. El primer cuestionario incluyó dos partes: La primera parte recogió datos sociodemográficos: edad, sexo, colegio, número de hermanos y la existencia de Al u OP. En la segunda parte se pidió a los padres que hicieran una descripción de los Al u OP si existían. Un segundo instrumento utilizado fueron las historietas (ver anexo 1).

El tercer instrumento que fue más bien una técnica, fue el sociograma en el cual los niños elegían al niño o niña con el que querían jugar. Esto se utilizó para conocer la popularidad de los niños en su contexto escolar.

\section{Procedimiento}

Con el fin de perfeccionar el diseño de la investigación y determinar el tipo de historias que debían utilizarse, se aplicaron las 4 historias de Harris y colaboradores (1986) y se realizó un estudio piloto con 15 niñas entre 4 y 6 años de un colegio privado de la ciudad de Bogotá. Este primer acercamiento permitió modificar las historias, reducirlas de 4 a 2 e incluir dibujos que facilitaran la comprensión y las respuestas de los niños y niñas. Los resultados obtenidos en este pilotaje no fueron incluidos en esta investigación. Las historias utilizadas se encuentran en el Anexo I.

Posteriormente y una vez modificados los instrumentos, se seleccionaron cinco escuelas infantiles privadas de un barrio de clase media alta de Bogotá. La selección se hizo por conveniencia, teniendo en cuenta que en dicha zona existen muchos jardines de infancia donde es posible encontrar niños de las edades del criterio de inclusión de la muestra. Una vez enviados y devueltos los cuestionarios destinados a los padres, los niños participantes fueron clasificados en función de la presencia de Al, de OP o de ninguno de los dos. En una segunda fase se realizaron las entrevistas con los niños en las que se presentaban las historias de comprensión emocional. En esta fase se aplicaron de manera individual $(N=43)$ los instrumentos a cada niño una vez se tenía claridad de si eran niños con Al (Amigos Imaginarios), niños con OP (Objetos Personificados) o niños sin Al ni OP.

El procedimiento consistió en ubicar al niño en el aula de clase, y de forma individual explicarle el procedimiento y aplicarle los instrumentos. La primera historia hacía referencia a una emoción aparente negativa en la que el protagonista era 
un niño o niña de su misma edad y la otra hacía referencia a una emoción aparente positiva en la que ellos eran los protagonistas (Ver anexo 1). Los niños escuchaban cada historia y luego debían repetirla para saber si la habían comprendido bien. En caso de tener errores de comprensión, se les volvía a leer, hasta que la comprendieran completamente.

Las entrevistas fueron grabadas y se utilizó un formato de calificación para clasificar las respuestas de los niños. La calificación de las respuestas correspondía a una escala de 0 y 1 . Los niños obtenían un 1 cuando su respuesta era correcta y la justificaban de forma coherente y 0 cuando la respuesta era incorrecta o no la explicaba de forma adecuada. En cada una de las dos historias, los niños obtenían una puntuación máxima de 2 si identificaban las emociones que se ocultaban y explicaban las razones por las cuales se ocultaban dichas emociones. Por ejemplo, para la segunda historia el niño obtenía una puntuación de 2 si lograba explicar que, para obtener el permiso de su madre para salir a jugar, debía aparentar una emoción que no sentía, diferenciando entre sus sentimientos reales y aparentes.

Una vez finalizada la presentación de las historias se pedía al niño que escogiera entre sus compañeros de clase aquél con el que más le gustaba jugar. Al final de la entrevista se entregó a cada niño un obsequio.

\section{Resultados}

Los objetivos del estudio se dirigieron a determinar las diferencias entre los niños con Al, con OP y los niños sin Al/OP con respecto a su nivel de popularidad y la capacidad para comprender las emociones aparentes en otros y en sí mismos. Los resultados fueron analizados con el programa SPSS. 22, basados en el análisis de varianzas (ANOVA) entre los tres grupos (Al, OP y sin Al/OP) con respecto a la comprensión de las emociones aparentes positivas en terceros, determinó que no existían diferencias significativas entre los tres grupos $(F(0,49=0.49$; $p>0.05$ ). Un segundo análisis utilizando las pruebas no - paramétricas y con el fin de disminuir los posibles problemas relacionados al tamaño de la muestra, corroboraron dichos resultados. La Tabla 1 presenta los resultados obtenidos.

Las diferencias entre grupos respecto a la comprensión de las emociones aparentes positivas en terceros no fueron significativas. Sin embargo, se muestra una tendencia, que si bien no es significativa, sugiere algunos elementos de análisis donde los niños con OP tienden a comprender mejor las situaciones relacionadas con emociones aparentes positivas.

Con respecto a la comprensión de emociones negativas en sí mismos se observa que no se presentaron diferencias significativas $(F=0.491 ; p>0.05)$ (Tabla 2). Sin embargo, las puntuaciones son un

Tabla1.

Promedios obtenidos en la comprensión de emociones aparentes en terceros

\begin{tabular}{cccc}
\hline Al & OP & Sin Al / OP & Significancia \\
\hline 0.54 & 0.80 & 0.47 & 0.492 \\
\hline
\end{tabular}

* $p<0.05$

Fuente: elaboración propia.

Tabla 2.

Promedios obtenidos en la comprensión de emociones aparentes negativas en sí mismos

\begin{tabular}{cccc}
\hline Al & OP & Sin Al / OP & Significancia \\
\hline 0.77 & 0.73 & 1 & 0.606 \\
\hline
\end{tabular}

${ }^{*} p<0.05$

Fuente: elaboración propia. 
poco mayores, comparativamente con los resultados obtenidos en los anteriores análisis, alcanzando el $n$ el caso de los niños sin Al ni OP. En la Tabla 2 se muestran los promedios obtenidos por los niños en la segunda historia.

En la Tabla 3 se presenta la tendencia de los promedios de los tres grupos respecto a la comprensión de emociones aparentes negativas en sí mismos. Una segunda hipótesis consideraba que los niños con Al tendrían un alto nivel de popularidad dentro de su grupo de compañeros. Los resultados muestran que los niveles de popularidad de los niños no fueron elevados si se tiene en cuenta que la puntuación máxima obtenida por uno de los niños fue 5 . El promedio más bajo fue obtenido por el grupo de niños con OP como se observa en la Tabla 3.

Con el fin de conocer si existían diferencias entre los grupos respecto a la comprensión de emociones aparentes positivas y negativas según la edad se analizaron los datos utilizando una prueba $t$ de student para muestras independientes y segmentando la muestra según el tipo de imaginación existente. El análisis de los resultados comparativos por edad a nivel general no mostró diferencias respecto a los grupos de edad en la comprensión de emociones aparentes positivas en terceros ni en emociones aparentes negativas en sí mismos. Sin embargo, si se muestran diferencias significativas respecto a la popularidad en función de la edad siendo más populares los niños mayores, como se observa en la Tabla 4.

El análisis segmentado por edades permitió determinar que los niños de 4 años con Al comprendían peor las emociones aparentes negativas en sí mismos $(M=0.20)$ que los niños de 5 años con Al $(M=0.75)$. Estas diferencias fueron estadísticamente significativas. Por el contrario, el grupo de niños con OP no presentó diferencias significativas en ninguno de los dos tipos de comprensión de emociones aparentes ni en la variable popularidad. Sin embargo, es importante resaltar que del grupo de niños con OP, los niños de 4 años obtuvieron una puntuación mayor que los de 5 años en la comprensión de emociones positivas en terceros. En el grupo de niños sin Al/OP tampoco se presentaron diferencias entre los grupos de edades respecto a la comprensión de emociones aparentes positivas o negativas. En la tabla 5 se muestran los promedios obtenidos por los niños de 3-4 años en la comprensión de emociones aparentes positivas y negativas y en el nivel de popularidad. Aún cuando existen diferencias entre los grupos, estas no fueron significativas.

Tabla 3.

Comparación de medias respecto a los resultados del sociograma

\begin{tabular}{cccc}
\hline Al & OP & Sin Al / Sin OP & Significancia \\
\hline 1.08 & 0.80 & 1.13 & 0.768 \\
\hline
\end{tabular}

${ }^{*} p<0.05$

Fuente: elaboración propia.

Tabla 4.

Comprensión de las emociones aparentes según la edad

\begin{tabular}{lccc}
\hline & 3-4 años & 5-6 años & Significancia \\
\hline Comprensión de emociones aparentes positivas en otros & 0.57 & 0.68 & 0.663 \\
Comprensión de emociones aparentes negativas en si mismo & 0.71 & 0.95 & 0.320 \\
Sociograma & 0.57 & 1.41 & $0.034^{* *}$ \\
\hline
\end{tabular}

${ }^{*} p<0.05$

Fuente: elaboración propia. 
Como un tercer nivel de análisis se determinaron algunos aspectos de las relaciones de los niños con su Al o con su OP partiendo de las descripciones realizadas por sus padres. De forma general se encontró que la mayoría de los niños disfrutan de la relación con sus Al u OP, los tratan como si en realidad tuvieran sentimientos y toman en cuenta lo que podrían sentir en ciertas circunstancias. La duración de esta relación suele variar entre 6 meses y un año.

En la Tabla 6 se presentan algunas de las características de los niños con Al y OP. En general los resultados indican que los niños con Al no son diferentes a los demás respecto a su popularidad ni a la comprensión de emociones aparentes en sí mismos ni en otros. Los niños mantienen una relación cordial y pacífica con sus amigos imaginarios u objetos personificado en presencia de otros, no siempre cuando están solos. En la discusión se ampliarán algunos elementos de análisis que podrían contemplarse en futuras investigaciones.

\section{Discusión}

Según los resultados encontrados, los niños con Al u OP no muestran diferencias significativas al compararlos con un grupo de niños sin Al/OP. Por lo tanto, los niños con Al u OP no muestran un mayor nivel de comprensión de las emociones aparentes, que los niños sin Al/OP. Estos datos concuerdan con los obtenidos por Harris y colaboradores (1986). Como se mencionó en la introducción, los supuestos acerca de la relación entre la imaginación de los niños y la comprensión de las emociones aparentes se basan en el papel de la imaginación (Harris et al., 1986) y en los estudios que encuentran que los niños con Al se desempeñan mejor en tareas de teoría de la mente (Taylor, 1999).

Autores como Harris y colaboradores (1986) o Taylor (1999) indican que los amigos imaginarios y los objetos personificados, actúan como simuladores de la realidad que permiten a los niños lograr practicar

Tabla 5.

Comprensión de las emociones aparentes en niños de 3-4 años

\begin{tabular}{lccc}
\hline & Al & OP & Al/OP \\
\hline Emociones Positivas & 0.20 & 1.0 & 0.29 \\
Emociones Negativas & 0.20 & 0.67 & 1.14 \\
Popularidad & 0.60 & 0.67 & 0.43 \\
\hline
\end{tabular}

${ }^{*} p<0.05$

Fuente: elaboración propia.

Tabla 6.

Características de las relaciones de los niños con sus Al y OP

\begin{tabular}{lcc}
\hline & OP & Al \\
& $n=15$ & Más de 6 meses \\
Tiempo de existencia en la vida del niño. & Más de 6 meses & $76 \%$ \\
Tiene en cuenta los sentimientos. & $93 \%$ & $76 \%$ \\
El personaje aparece cuando el niño está sólo. & $73 \%$ & $23 \%$ \\
El niño se muestra muy feliz cuando está en su compañía. & $6 \%$ & $100 \%$ \\
\hline
\end{tabular}

Fuente: elaboración propia. 
su capacidad de comprensión de las creencias, intenciones y sentimientos de los otros. Además, estas capacidades les permitirían a los niños ser más sociables debido a una capacidad más desarrollada en comprender los estados de ánimo de los demás, así como sus creencias.

Así pues, los niños con Al o con OP deberían comprender mejor que los demás las emociones aparentes. Sin embargo, los datos reportados no apoyan este planteamiento. Como se mencionó, los estudios sobre la comprensión de emociones aparentes mostraban que los niños de 4 años presentaban muchas dificultades para comprenderlas, mientras que los niños de 6 años ya las entendían claramente (Harris et al., 1986). En este sentido, al retomar los datos obtenidos en este estudio respecto a las diferencias entre edades, no se presentaron diferencias significativas entre los grupos de 3-4 y 5-6 años en el nivel de comprensión de las emociones aparentes tanto positivas como negativas. En general, los promedios obtenidos por los grupos no fueron altos y casi no superaron la media. Estos resultados muestran que el ejercicio de comprensión de las emociones aparentes es difícil para los niños de estas edades y que su comprensión es similar para aquellos de 4, 5 o 6 años.

En segundo lugar, los niños con Al u OP no son evaluados por su grupo de iguales como mejores compañeros de juego, es decir no lograron mayores niveles de popularidad. Si bien los estudios de Manosevitz y colaboradores (1973), planteaban una tendencia importante de los niños con Al a ser sociables con los adultos, no necesariamente sucede lo mismo con el grupo de compañeros. Si bien ya se ha considerado que los datos de los estudios con niños con Al suelen ser contradictorios entre sí, nuestros resultados tampoco apoyan los planteamientos de Harter y Chao (1992), quienes mostraron que los niños con Al eran menos populares y menos aceptados socialmente por su grupo de compañeros que aquellos sin Al.

En este sentido, nuestros datos apoyan las posturas críticas y los planteamientos de Taylor (1999) quien considera que los niños con Al no son ni más ni menos inteligentes, sociables, creativos o alegres que los demás niños de su edad. Queda entonces sin resolver una importante cuestión: ¿por qué determinados niños inventan personajes con los que mantienen una estrecha relación si no difieren de sus compañeros en rasgos de personalidad o aspectos como la sociabilidad? Aunque la investigación no responde a esta cuestión, parece un interesante aspecto a retomar en el futuro. Quizás elementos externos como el contexto social podrían ayudar a la explicación. En este sentido, una de las variables que claramente parece tener relación con la aparición de Al es la estructura familiar que, aunque no daría cuenta de forma completa del fenómeno, puede ser un elemento importante a investigar.

Si bien los resultados no apoyan las hipótesis del estudio, los datos obtenidos son coherentes con las posturas de Dunn (1995) y Hay y colaboradores (2004), quienes plantean una estrecha relación entre la comprensión de las emociones ajenas y la popularidad de los niños en su grupo de iguales. Los niños de este estudio no lograron niveles elevados de comprensión de las emociones aparentes, ajenas ni propias, pero tampoco lograron niveles elevados de popularidad dentro de su grupo de iguales.

Dos explicaciones podrían dar cuenta de los resultados obtenidos en este estudio. Por una parte, es posible que simplemente la tarea presentada haya resultado demasiado compleja para los participantes. Por otra, quizás la imaginación no constituya una variable relevante a la hora de explicar la comprensión de las emociones aparentes. A pesar de la sensatez de la propuesta de Harris $(1989,2000)$, es posible que la comprensión de las emociones aparentes dependa en primer lugar de otras capacidades cognitivas, como la capacidad representacional, el espacio mental, o la capacidad atencional, más que de una cuestión imaginativa.

Además, existen ciertos aspectos procedimentales que es necesario señalar. En primer lugar, el reducido número de niños entrevistados no permite generalizar los datos obtenidos. Aunque la muestra alcanzó los 43 niños en total, es cierto que los grupos de comparación quedaban reducidos a 13 o 15 niños. En segundo lugar, uno de los principales problemas, que también aborda Taylor (1999), hace 
referencia a la clasificación de los niños y a la comprobación de la existencia de Al. Usualmente se ha utilizado a los padres como fuente de información, pero ellos no siempre tienen a la información completa sobre la vida mental de sus hijos ni sobre la existencia de los Al. Muchos padres desconocen la existencia de Al en la vida de sus hijos o confunden un juego simbólico con un Al. En el caso de los OP la detección de los casos es más sencilla, puesto que los objetos son claramente visibles por varias personas y los niños suelen llevarlos consigo a todas partes. Este problema de detección de casos es muy relevante para nuestro estudio. Dado que no se pudo entrevistar a los padres con detenimiento y que no se plantearon preguntas directas sobre los Al a los propios niños, es posible que algunos casos no correspondan exactamente con la definición que hemos ofrecido de amigo imaginario. Para futuras investigaciones sería fundamental realizar entrevistas en profundidad con los padres y con los niños para determinar con mayor certeza la existencia de un Al.

Por último, para que la medida de la popularidad resultara más fiable podría también modificarse mediante la inclusión de todos los niños de la clase, no sólo de aquellos que participaron en la investigación. Respecto a las relaciones de los niños con sus Al y OP se encontró que suelen ser muy positivas y de amistad, como lo planteaba Gleason y colaboradores (2000). Los niños suelen estar atentos a las necesidades y sentimientos de sus Al u OP y comparten con su familia esta preocupación. Las respuestas de los padres permitieron determinar que los niños interactúan con sus Al y OP en presencia de otros y los involucran en sus actividades cotidianas.

Finalmente, este estudio aportó un análisis diferencial de las capacidades de los niños con Al y OP respecto a la comprensión de las emociones aparentes y su popularidad. Esta diferenciación entre los dos tipos de compañeros que crean los niños es un avance metodológico importante que ya ha sido contemplado por varios autores (Gleason et al., 2000; Manosevitz et al., 1973; Taylor, 1999), quienes defienden la creación de categorías independientes para nombrar cada uno de los tipos de imaginación infantil.

\section{Referencias}

Ames, L. B. y Learned, J. (1946). Imaginary companions and related phenomena. The Pedagogical Seminary and Journal of Genetic Psychology, 69(2), 147-167. https://doi.org/10.1080/088565 59.1946.10533385.

Carter, C. y Bath, C. (2018). The pirate in the pump: children's views of objects as imaginary friends at the start of school. Education 3(13), 335-344. https://doi.org/10.1080/03004279.2016.1262887

Dunn, J. (1995). Children as psychologist: The later correlates of individual difference in understanding of emotions and other minds. Cognition and Emotion, 9(2-3), 87-201. https:// doi.org/10.1080/02699939508409008

Galyer, K. y Evans, I. (2001). Pretend play and the development of emotion regulation in preschool children. Early Child Development and Care, 166, 93-108. https://doi. org/710.1080/0300443011660108.

Gleason, T. R. (2002). Social provisions of real and imaginary relationships in early childhood. Developmental Psychology, 38(6), 979-992. https://doi.org/10.1037/0012-1649.38.6.979.

Gleason, T. R. y Kalpidou, M. (2014). Imaginary companions and young children's coping and competence. Social Development, 23(4), 820-839. http://dx.doi.org/10.1111/sode.12078

Gleason, T. R., Sebanc, A. M. y Hartup, W. W. (2000). Imaginary companions of preschool children. Developmental Psychology, 36(4), 419-428. https://doi.org/10.1037/0012-1649.36.4.419

Harris, P., Donnelly, K., Guz, G. y Pitt-Watson, R. (1986). Children's understanding of the distinction between real and apparent emotion. Child Development, 57(4), 895-909. https://doi. org/10.2307/1130366

Harris, P. (1989). Los Niños y las Emociones. Madrid: Alianza Psicología Minor.

Harris, P. (2000). The Work of Imagination. Oxford: Blackwell Publishers. 
Harter, S. y Chao, C. (1992). The role of competence in children's creation of imaginary friends. Merrill-Palmer Quarterly, 38(3), 350-363.

Hay, D. F., Payne, A. y Chadwick, A. (2004). Peer relations in childhood. Journal of Child Psychology and Psychiatry, 45(1), 84-108. https://doi. org/10.1046/j.0021-9630.2003.00308.x

Lin, Q., Fu, H., Wan., Zhou, N. y Xu, H. (2018). Chinese children's imaginary companions: Relations with peer relationships and social competence. International Journal of Psychology, 53(5), 388-396. https://doi.org/10.1002/ijop.12392

Moore, R. y Russ, S. (2006). Pretend play as a resource for children: implications for pediatricians and health professionals. Journal of Developmental Behavioral Pediatrics 27(3), 237-248. https:// doi.org/10.1097/00004703-200606000-00011

Manosevitz, M., Fling, S. y Prentice, N. (1977). Imaginary companions in young children: Relationships with intelligence, creativity and waiting ability. Journal of Child Psychology and Psychiatry, 18(1), 73-78. https://doi. org/10.1111/j.1469-7610.1977.tb00418.x.

Manosevitz, M., Prentice, N. y Wilson, F. (1973). Individual and family correlates of imaginary companions in preschool children. Developmental Psychology, 8(1), 72-79. https:// doi.org/10.1037/h0033834.

Moriguchi, Y., Shinohara, I. y Ishibashi, M. (2016). Agent perception in children with and without imaginary companions. Infant and Child Development, 25(6), 550-564. https://doi. org/10.1002/icd.1953.

Moriguchi, Y. y Todo, N. (2019). Prevalence of imaginary companions in Japanese children. International Journal of Psychology, 54(2), 269276. https://doi.org/10.1002/ijop.12446.
Pearson, D., Rouse, H., Doswell, S., Ainsworth., C., Dawson, O., Simms, K., Edwards, L.y Faulconbridge, J. (2001). Prevalence of imaginary companions in a normal child population. Child: Care, Health and Development, 27(1), 13-22. https://doi. org/10.1046/j.1365-2214.2001.00167.x

Russ, S. (2004). Play in child development and psychotherapy: Toward empirically supported practice. Washington: Lawrence Erlbaum Associates Publishers.

Singer, D. G. y Singer, J. L. (1990). The house of make-believe: Children's play and the developing imagination. Cambridge, MA: Harvard University Press.

Singer, J. L. (1961). Imagination and waiting ability in young children. Journal of Personality, 29, 396-413. http://doi. org/10.1111/j.1467-6494.1961.tb01670.x.

Svendsen, M. (1934). Children's Imaginary Companions. Archives of Neurology and Psychiatry, 32(5), 985-999. https://doi.org/10.1001/ archneurpsyc.1934.02250110073006

Taylor, M. (1999). Imaginary Companions and the Children Who Create Them. Oxford: Oxford University Press.

Taylor, M. y Carlson, S. M. (1997), The relation between individual differences in fantasy and theory of mind. Child Development, 68, 436-455. https:// doi.org/10.1111/j.1467-8624.1997.tb01950.x

Taylor, M., Cartwright, B. S. y Carlson, S. M. (1993). A developmental investigation of children's imaginary companions. Developmental Psychology, 29(2), 276-285. https://doi. org/10.1037/0012-1649.29.2.276. 


\section{Anexo I}

\section{Historietas. (Dirigida a las niñas)}

Diana tiene tu misma edad. A ella no le gusta ir donde su abuelito porque se aburre mucho. Diana no quiere que el abuelito se dé cuenta de sus sentimientos y entonces trata de esconderlos para no hacerlo sufrir.

\section{Historietas. (Dirigida a los niños)}

Mario tiene tu misma edad. A el no le gusta ir donde su abuelito porque se aburre mucho. Mario no quiere que el abuelito se dé cuenta de sus sentimientos y entonces trata de esconderlos para no hacerlo sufrir.

¿Por favor me repites la historia? (si el niño no ha comprendido bien la historia se vuelve a contar hasta que la comprenda).

\section{Preguntas}

1. ¿Qué cara crees que pone Diana o Mario si la pregunta se dirige al niño, cuando saluda a su abuelito? Por favor señala una de estas caras

2. ¿Por qué crees que pone esa cara?

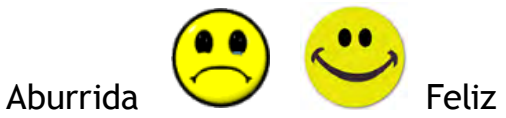

3. ¿Qué cara crees que pone Diana (Mario si estamos hablando a un niño), cuando el abuelito no la (lo) está viendo?

4. ¿Por qué crees que pone esa cara?

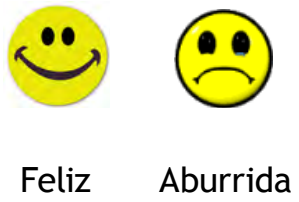

\section{Historia 2}

¿Alguna vez has estado enferma (o)? Bueno pensemos que estás enferma (o) en tu casa y quieres salir a jugar con tus amigos. Tú sabes que si tu mamá se da cuenta que estás enfermo (a) no te va a dejar salir a jugar. Como tú quieres salir a jugar entonces vas a aparentar que estas sana (o).

¿Por favor me repites la historia?

Cuando el niño o la niña repiten la historia y se puede determinar que comprendieron entonces se les pregunta:

1. ¿Qué cara pusiste cuando le pediste permiso a tu mamá para salir?

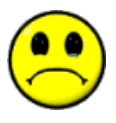

Sana

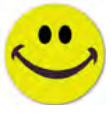

Enferma
¿Por qué pusiste esa cara?

¿Cómo te sentías de verdad?

¿Por qué te sentías así?

\section{Sociograma}

Después de narrar las historias, se le pidió a los niños entrevistados que señalaran entre sus compañeros el más popular. Es decir aquel con el que siempre quieren jugar y les cae muy bien.

Se les pregunto

¿Cuál es el o la niña de tú clase con quien más te gusta estar? 